\title{
ACCURATE BER OF MC-DS-CDMA OVER RAYLEIGH FADING CHANNELS
}

\author{
Besma Smida \\ Division of Engineering and Applied Sciences, \\ Harvard University, Cambridge, USA \\ bsmida@deas.harvard.edu \\ Lajos Hanzo \\ School of Electronics and Computer Science, \\ University of Southampton, UK \\ Ih@ecs.soton.ac.uk \\ Sofiène Affes \\ INRS-EMT, \\ Université du Québec, Canada \\ affes@emt.inrs.ca
}

Abstract In this paper, we derive an accurate average bit error rate (BER) of Rayleighfaded MC-DS-CDMA in the context of asynchronous transmission and random spreading sequences. The analysis is based on the characteristic function, and does not resort to any assumption on the statistical behavior of the interference. We develop a new closed-form expression for the conditional characteristic function of the inter-carrier interference and a single integration for the BER calculation. The accuracy of the standard Gaussian approximation (SGA) method is also evaluated.

Keywords: multi-carrier direct-sequence CDMA (MC-DS-CDMA), performance evaluation, characteristic function

\section{Introduction}

As a combination of multi-carrier (MC) modulation and direct-sequence code-division multiple-access (DS-CDMA), MC-DS-CDMA [1] benefits from both techniques. First, the parallel transmission nature of multi-carrier modulation makes it especially attractive for broadband communications [2]. Secondly, with advanced signal processing techniques, MC-DS-CDMA has the 
potential to provide larger capacity than other multiple access schemes. Biterror rate (BER) is one of the most important performance measures for communication systems that has been studied extensively. The multiple access interference (MAI) and the inter-symbol interference (ISI), which are inherited from conventional DS-CDMA, affect likewise the performance of MCDS-CDMA systems. In addition, MC-DS-CDMA capacity is limited by the inter-carrier interference (ICI) due to the use of multicarrier modulation. To analyze the BER performance of MC-DS-CDMA systems, the interferences MAI, ISI and ICI are commonly assumed to be Gaussian distributed [1][4]. However, the accuracy of the Gaussian approximation techniques depends on the specific configuration of the system. It is well known that the Gaussian approximation techniques become less accurate, when a low number of users is supported or when there is a dominant interferant [5].

Therefore the accurate BER analysis dispensing with the previous assumptions on the interference distribution is desirable. To avoid the Gaussian approximation of the interference, the BER can be calculated in transform domains. Two widely used transforms are the Fourier and Laplace transforms, corresponding to the characteristic function $(\mathrm{CF})$ and the moment generating function (MGF), respectively. The basic idea is that first the CF or MGF of the decision variable is derived, then an associated inverse transform is performed to calculate the BER. Since the decision variable can be completely and exactly characterized by its CF or MGF, the BER can be accurately evaluated via numerical integration techniques. For this reason, CF- and MGF-based methods have received considerable attention. The CF method was used to study the BER performance of DS-CDMA with random sequences in Rayleigh [6] and Nakagami-fading [7] channels. A saddle point integration-based MGF approach was proposed to compute the error probabilities of DS-CDMA systems over Rician [8] and Nakagami-fading [9] channels. This approach has been applied to study the performance of MC-DS-CDMA systems with deterministic spread sequences [10]. However, to the author's best knowledge, the accurate BER analysis of asynchronous Rayleigh-faded MC-DS-CDMA using random sequences is still an open problem. In this paper, we will derive an accurate BER of Rayleigh-faded MC-DS-CDMA in the context of asynchronous transmission and random spreading sequences. The analysis is based on the $\mathrm{CF}$, and does not resort to any assumption on the statistical behavior of the interference. A new closed-form expression, rather than an integral [10], is derived for the conditional characteristic function of the inter-carrier interference.

\section{System and Channel Model}

We consider an asynchronous Rayleigh-faded MC-DS-CDMA system using BPSK modulation, random spreading sequences and rectangular chip wave- 
forms. The input information sequence of the $k$-th user is first converted into $U$ parallel data sequences $b_{u}^{k}(t)$ for $u=1,2, \ldots, U$. The data sequence $b_{u}^{k}(t)=\sum_{i=-\infty}^{\infty} b_{u, i}^{k} P_{T_{s}}\left(t-i T_{s}\right)$ consists of a sequence of mutually independent rectangular pulses of duration $T_{s}$ and of amplitude +1 or -1 with equal probability. After serial-to-parallel conversion, the $u$-th substream modulates a subcarrier frequency $f_{u}$ using binary phase shift keying (BPSK). Then, the $U$ modulated subcarriers are added in order to form the complex modulated signal. Finally, spectral spreading is imposed on the complex signal by multiplying it with a spreading code. Therefore, the transmitted signal of the $k$-th user is given by:

$$
s^{k}(t)=\sum_{u=1}^{U} \sqrt{2 P} b_{u}^{k}(t) a^{k}(t) \cos \left(j 2 \pi f_{u} t+\phi_{u}^{k}\right),
$$

where $P$ represents the transmitted power per subcarrier, while $a^{k}(t)$ and $\phi_{u}^{k}$ represent the spreading-code segment and the phase angle introduced in the carrier modulation process. The spreading sequence can be expressed as $a^{k}(t)=$ $\sum_{l=-\infty}^{\infty} a_{l}^{k} P_{T_{c}}\left(t-i T_{c}\right)$, where $a_{l}^{k}$ assumes values of +1 or -1 with equal probability, while $P_{T_{c}}(t)$ is the rectangular chip waveform that is defined over the interval $\left[0, T_{c}\right) . T_{c}=\frac{T_{s}}{L}$ is the chip duration, while $L$ is the spreading factor.

For MC-DS-CDMA, the modulated subcarriers are orthogonal over the chip duration. Hence, the frequency corresponding to the $u$-th subcarrier is $f_{u}=$ $f_{p}+u / T_{c}$, where $f_{p}$ is the fundamental carrier frequency.

We assume that the channel between the $k$-th transmitter and the corresponding receiver is a slowly flat Rayleigh fading channel over each subcarrier. The channel impulse response for the $k$-th transmitted signal over the $u$-th subcarrier is given by

$$
H_{u}^{k}(t)=h_{u}^{k} \delta\left(t-\tau_{k}\right) \exp \left(-j \varphi_{u}^{k}\right)
$$

where $h_{u}^{k}, \tau_{k}$ and $\varphi_{u}^{k}$ represent the attenuation factor, delay and phase-shift, respectively. The attenuations $h_{u}^{k}$ are independent Rayleigh variables, while $\varphi_{u}^{k}$ are i.i.d. random variables uniformly distributed in the interval $[0,2 \pi)$. The delay $\tau_{k}$ represents the user asynchronism and it is assumed to be uniform over $\left[0, T_{s}\right)$.

We consider $K$ asynchronous MC-DS-CDMA users in the system having all the same number of subcarriers $U$ and spreading factor $L$. The average power received from each user is also assumed to be the same. Consequently, the received signal may be written as:

$$
r(t)=\sum_{k=1}^{K} \sum_{u=1}^{U} \sqrt{2 P} h_{u}^{k} b_{u}^{k}\left(t-\tau_{k}\right) a^{k}\left(t-\tau_{k}\right) \cos \left(j 2 \pi f_{u} t+\varphi_{u}^{k}\right)+N(t),
$$


where $\varphi_{u}^{k}=\phi_{u}^{k}-\varphi_{u}^{k}-2 \pi f_{u} \tau_{k}$, which is assumed to be an i.i.d. random variable having a uniform distribution in $[0,2 \pi)$, while $N(t)$ represents the additive white Gaussian noise (AWGN) with zero mean and double-sided power spectral density of $N_{o} / 2$.

\section{BER Analysis}

\section{Decision variable statistics}

Consider using a conventional single-user matched filter to coherently demodulate the desired user signal. We assume, without loss of generality, the target user signal has index $k=1$ and $\tau_{1}=0, P=2$, and $T_{c}=1$. The decision variable of the first user over the $v$-th subcarrier is:

$$
\begin{aligned}
Z_{v} & =\int_{0}^{T_{s}} r(t) \cdot a^{1}(t) \cos \left(j 2 \pi f_{v} t+\varphi_{v}^{1}\right) d t \\
& =D_{v}+\sum_{k=2}^{K} I_{1}^{k}+\sum_{k=2}^{K} \sum_{u=1, u \neq v}^{U} I_{2}^{k}+N_{v}
\end{aligned}
$$

where

- $D_{v}=h_{v}^{1} b_{v, 0}^{1} L$ is the desired output.

- $I_{1}^{k}=h_{v}^{k} \cos \left(\theta_{v}^{k}\right) W^{k}$ is the interference due to the other users on the same carrier $v$, where $\theta_{v}^{k}=\varphi_{v}^{1}-\varphi_{v}^{k}$. In their notable work on random sequences for DS-CDMA, Lehnert and Pursley [11] simplified the expression of the random variable $W^{k}$. For a rectangular chip waveform, the random variable $W^{k}$ was further simplified by Cheng and Beaulieu in [6] as follows:

$$
W^{k}=P^{k} \nu_{k}+Q^{k}\left(1-\nu_{k}\right)+X^{k}+Y^{k}\left(1-\nu_{k}\right),
$$

where $\nu_{k}$ is a uniform $\operatorname{RV}$ over $[0,1), P^{k}$ and $Q^{k}$ are symmetric Bernoulli RVs. $X^{k}$ is a discrete RV that represents the sum of $A$ independent symmetric Bernoulli RVs and $Y^{k}$ is a discrete RV that represents the sum of $B$ independent symmetric Bernoulli RVs. Note that $A+B=L-1$ and the marginal pdf's of $X^{k}$ and $Y^{k}$ are given by:

$$
\begin{aligned}
P_{X^{k}}(j) & =\left(\begin{array}{c}
A \\
\frac{j+A}{2}
\end{array}\right) 2^{-A}, \\
j \in \mathcal{A} & =\{-A,-A+2, \ldots, A-2, A\}
\end{aligned}
$$


and

$$
\begin{aligned}
P_{Y^{k}}(j) & =\left(\begin{array}{c}
B \\
\frac{j+B}{2}
\end{array}\right) 2^{-B}, \\
j \in \mathcal{B} & =\{-B,-B+2, \ldots, B-2, B\} .
\end{aligned}
$$

- $I_{2}^{k}=h_{u}^{k} W_{u-v}^{k}$ is the inter-carrier interference due to the other carriers $(u \neq v)$ from the other users. We derive the random variable $W_{u-v}^{k}$ as follows:

$$
W_{u-v}^{k}=b_{u,-1}^{k} R_{k, 1, u-v}\left(\tau_{k}, \theta_{u}^{k}\right)+b_{u, 0}^{k} \hat{R}_{k, 1, u-v}\left(\tau_{k}, \theta_{u}^{k}\right),
$$

where $R_{k, 1, u-v}\left(\tau_{k}, \theta_{u}^{k}\right)$ and $\hat{R}_{k, 1, u-v}\left(\tau_{k}, \theta_{u}^{k}\right)$ are the partial cross-correlation functions defined by:

$$
\begin{aligned}
& R_{k, 1, u-v}\left(\tau_{k}, \theta_{u}^{k}\right)=\int_{0}^{\tau_{k}} a^{k}\left(t-\tau_{k}\right) a^{1}(t) \cos \left(2 \pi\left(f_{u}-f_{v}\right) t+\theta_{u}^{k}\right),(1 \\
& \hat{R}_{k, 1, u-v}\left(\tau_{k}, \theta_{u}^{k}\right)=\int_{\tau_{k}}^{T_{s}} a^{k}\left(t-\tau_{k}\right) a^{1}(t) \cos \left(2 \pi\left(f_{u}-f_{v}\right) t+\theta_{u}^{k}\right) .
\end{aligned}
$$

In this paper, we follow the same methodology used in [11] and use the formula (23) of [1] to simplify $W_{u-v}^{k}$ as

$$
\begin{aligned}
W_{u-v}^{k}= & \cos \left(\theta_{u}^{k}+\pi \nu_{k}|u-v|\right) \times \\
& {\left[\left(P^{k}-Q^{k}\right) \nu_{k} \operatorname{sinc}\left(\nu_{k}|u-v|\right)-2 Y^{k} \nu_{k} \operatorname{sinc}\left(\nu_{k}|u-v|\right)\right], } \\
= & \cos \left(\theta_{u}^{k}+\pi \nu_{k}|u-v|\right) \mathcal{W}_{u-v}^{k},
\end{aligned}
$$

where $\theta_{u}^{k}=\varphi_{v}^{1}-\varphi_{u}^{k}$ and $\left(\theta_{u}^{k}+\pi \nu_{k}|u-v|\right)$ is uniform RV over [0, $\left.2 \pi\right)$. Note that $X^{k}$ does not exit in the formulation of $W_{u-v}^{k}$ which will reduce the complexity of the BER calculation.

- $N_{v}$ is the noise, which is a zero-mean Gaussian random variable with a variance $\sigma_{n}=N_{o} L / 4$.

\section{Exact BER Analysis}

In this section, we will use the characteristic function method to compute the average BER under asynchronous transmission conditions in flat Rayleigh fading. The characteristic function of the interference $I_{1}^{k}$ due to the other users on the same carrier, $\Phi_{I_{1}^{k} \mid B}$, was derived by Eqs (31)-(32) in [6] as follows:

$$
\begin{aligned}
\Phi_{I_{1}^{k} \mid B}(w)= & \frac{2^{-(N-1)}}{4} \sum_{i \in A} \sum_{j \in B}\left(\begin{array}{c}
A \\
\frac{i+A}{2}
\end{array}\right)\left(\begin{array}{c}
B \\
\frac{j+B}{2}
\end{array}\right) \\
& \times\left[J_{1}(i+1, j)+J_{1}(i, j-1)+J_{1}(i, j+1)+J_{1}(i-1, j)\right](15)
\end{aligned}
$$


where

$$
J_{1}(i, j)=\left\{\begin{array}{ll}
\exp \left(-\frac{i^{2}}{2} w^{2}\right), & j=0 \\
\frac{\sqrt{\frac{\pi}{2}}}{j|w|}\{Q(|w|(i-j))-Q(|w|(i+j))\}, & (j, w \neq 0)
\end{array},\right.
$$

where $Q$ is the standard $Q$ function. In this paper we will derive the characteristic function of the inter-carrier interference $I_{2}^{k}$. We first examine the statistics of each interferer $I_{2}^{k}$. From the previous section, we have $I_{2}^{k}=$ $h_{u}^{k} \cos \left(\theta_{u}^{k}+\pi \nu_{k}|u-v|\right) \mathcal{W}_{u-v}^{k}$, where $h_{u}^{k} \cos \left(\theta_{u}^{k}+\pi \nu_{k}|u-v|\right)$ is a zeromean unit-variance Gaussian RV and $\mathcal{W}_{u-v}^{k}$ is defined in Eq. (14). This implies that $I_{2}^{k}$ given $P_{k}, Q_{k}, Y_{k}, \nu_{k}, B$ is Gaussian with zero mean and variance $\sigma_{I_{2}^{k} \mid \mathcal{W} k-v}^{2}=\left(\mathcal{W}_{u-v}^{k}\right)^{2}$. Averaging over $P_{k}, Q_{k}, Y_{k}$ (which is equivalent to averaging over all interferes' spreading sequences and data sequences), yields to the characteristic function of the inter-carrier interference $I_{2}^{k}$ given $\nu_{k}$ and $B$ as:

$\Phi_{I_{2}^{k} \mid \nu_{k}, B}(w)=\frac{2^{-B}}{4} \sum_{j \in B}\left(\begin{array}{c}B \\ \frac{J+B}{2}\end{array}\right)\left\{\sum_{l=1,2,3,4} \exp \left\{\frac{1}{2} \sigma_{l}^{2}\left(j, \nu_{k}, u-v\right) w^{2}\right\}\right\}$,

where

$$
\begin{aligned}
\sigma_{1}^{2}\left(j, \nu_{k}, u-v\right) & =\left[2 j \nu_{k} \operatorname{sinc}\left(\nu_{k}|u-v|\right)\right]^{2} \\
\sigma_{2}^{2}\left(j, \nu_{k}, u-v\right) & =\left[2 j \nu_{k} \operatorname{sinc}\left(\nu_{k}|u-v|\right)\right]^{2} \\
\sigma_{3}^{2}\left(j, \nu_{k}, u-v\right) & =\left[2(1-j) \nu_{k} \operatorname{sinc}\left(\nu_{k}|u-v|\right)\right]^{2} \\
\sigma_{4}^{2}\left(j, \nu_{k}, u-v\right) & =\left[2(1+j) \nu_{k} \operatorname{sinc}\left(\nu_{k}|u-v|\right)\right]^{2} .
\end{aligned}
$$

The $\nu_{k}$ 's now appear in the numerators of the exponential function and averaging can be carried out by using Eq. (3.339) in [12] to give the characteristic function of $I_{2}^{k}$ given $B$ as:

$$
\begin{aligned}
\Phi_{I_{2}^{k} \mid B}(w) & =\int_{0}^{1} \Phi_{I_{2}^{k} \mid \nu_{k}, B}(w) d \nu_{k} \\
& =\frac{2^{-B}}{4} \sum_{j \in B}\left(\begin{array}{c}
B \\
\frac{j+B}{2}
\end{array}\right) \times[2 J(j)+J(j-1)+J(j+1)]
\end{aligned}
$$

where $B$ varies from 0 to $L-1$, and

$$
J(j)=\exp \left(-\frac{j^{2}}{\pi^{2}(u-v)^{2}} w^{2}\right) I_{0}\left(-\frac{j^{2}}{\pi^{2}(u-v)^{2}} w^{2}\right) .
$$


$I_{0}$ is the modified Bessel function of order 0 . Hence the characteristic function of the total interference is given by:

$$
\Phi_{I \mid B}(w)=\Pi_{k=2}^{K}\left[\Phi_{I_{1}^{k} \mid B}(w) \Pi_{u=1, u \neq v}^{U} \Phi_{I_{2}^{k} \mid B}(w)\right] .
$$

The conditional BER for a Rayleigh fading channel can be expressed by:

$$
\begin{aligned}
P_{e \mid B}= & \frac{1}{2}\left[1-\frac{L}{\sqrt{L^{2}+\sigma_{n}^{2}}}\right]+\frac{L}{\sqrt{2 \pi}} \int_{0}^{+\infty}\left[1-\Phi_{I \mid B}(w)\right] \Phi_{n}(w) \\
& \times \exp \left\{-\frac{1}{2} w^{2} L^{2}\right\} d w,
\end{aligned}
$$

where $\sigma_{n}^{2}$ is the variance of the background noise. Finally, the overall average BER is obtained by averaging $P_{e \mid B}$ over all spreading sequences, yielding:

$$
P_{e}=2^{-(L-1)} \sum_{B=0}^{L-1}\left(\begin{array}{c}
L-1 \\
B
\end{array}\right) P_{e \mid B} .
$$

\section{Standard Gaussian Approximation}

Using the derivations found in [3], the average BER $P_{e}$ in Rayleigh fading approximated by the SGA can be shown to be:

$$
P_{e} \approx \frac{1}{2}\left[1-\frac{1}{\sqrt{1+\frac{N_{o}}{4 L}+\gamma}}\right]
$$

where

$$
\gamma=\frac{2(K-1)}{3 L}+(K-1)\left[\frac{1}{U} \sum_{u=1}^{U} \sum_{u=1, u \neq v}^{U} \frac{1}{\pi^{2}(u-v)^{2} L}\right] .
$$

\section{Numerical Results}

In this section we will compare our simulation results to those obtained from our accurate BER analysis as well as to those generated by the SGA. Since the evaluation of the effect of the ICI on the performance of MC-DSCDMA is the main objective of our analysis, we assumed first that the effect of noise is negligible. Fig. 1 shows the average BER performance against the number of users. The accuracy of our BER expression was confirmed by simulations. On the other hand, the SGA slightly over-estimates the BER. The inaccuracy of the SGA becomes prevalent when the number of users, the spreading factor, and/or the number of subcarriers decreases. Fig. 2 illustrates 
the average BER performance versus the per-bit SNR, when the number of users is $K=4$. It compares the results obtained by the SGA to our accurate BER. Fig. 2 confirms, not surprisingly, that the presence of strong background noise improves the accuracy of the SGA method.

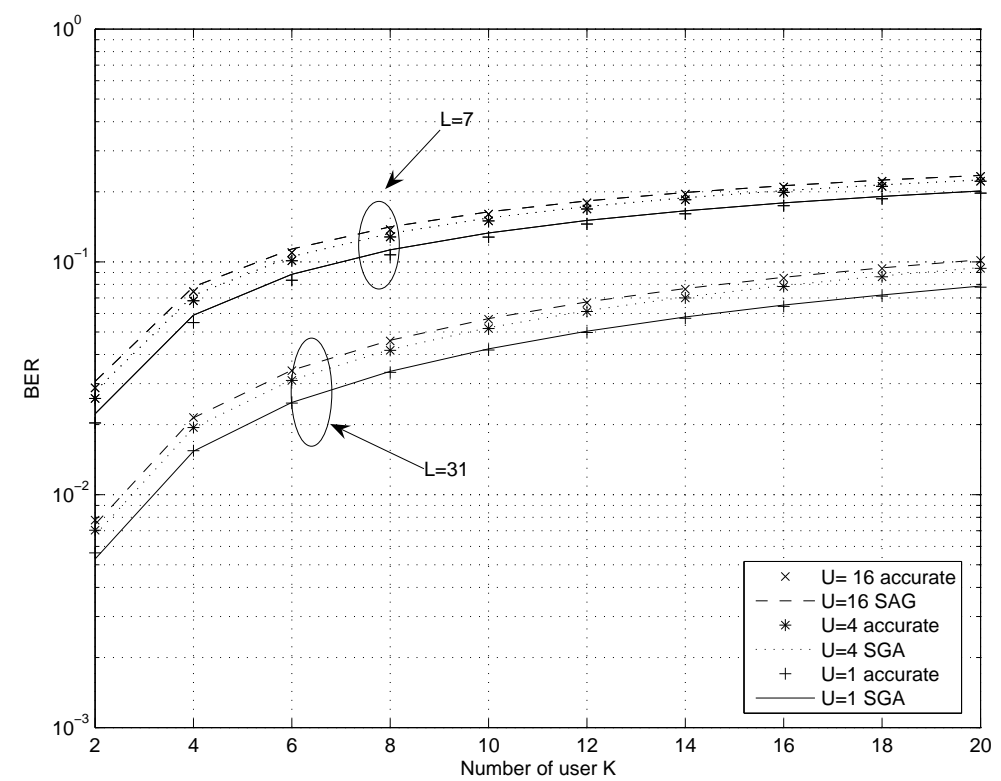

Figure 1. BER vs the number of users $K$ in an asynchronous MC-DS-CDMA exposed to Rayleigh fading with random spreading sequences and BPSK modulation. The length of the spreading sequence is $L=7$ and $L=31$. The number of subcarriers is $U=1,4$, and 9 . The average power of all subcarriers and users is equal and the background noise is ignored.

\section{Conclusion}

We studied the accurate BER calculation of an asynchronous MC-DS-CDMA system exposed to flat Rayleigh fading using random spreading sequences and BPSK modulation. Using the CF approach, we derived a new closed-form expression for the conditional characteristic function of the inter-carrier interference and a single integration for the BER calculation. The inaccuracy of the SGA becomes prevalent when the number of users, the spreading factor, and/or the number of subcarriers decreases.

\section{References}

[1] E.A. Sourour and M. Nakagawa, "Performance of orthogonal multicarrier CDMA in a multipath fading channel," IEEE Transactions on Communications, vol. 44, no. 3, pp. 356367, Mar. 1996. 


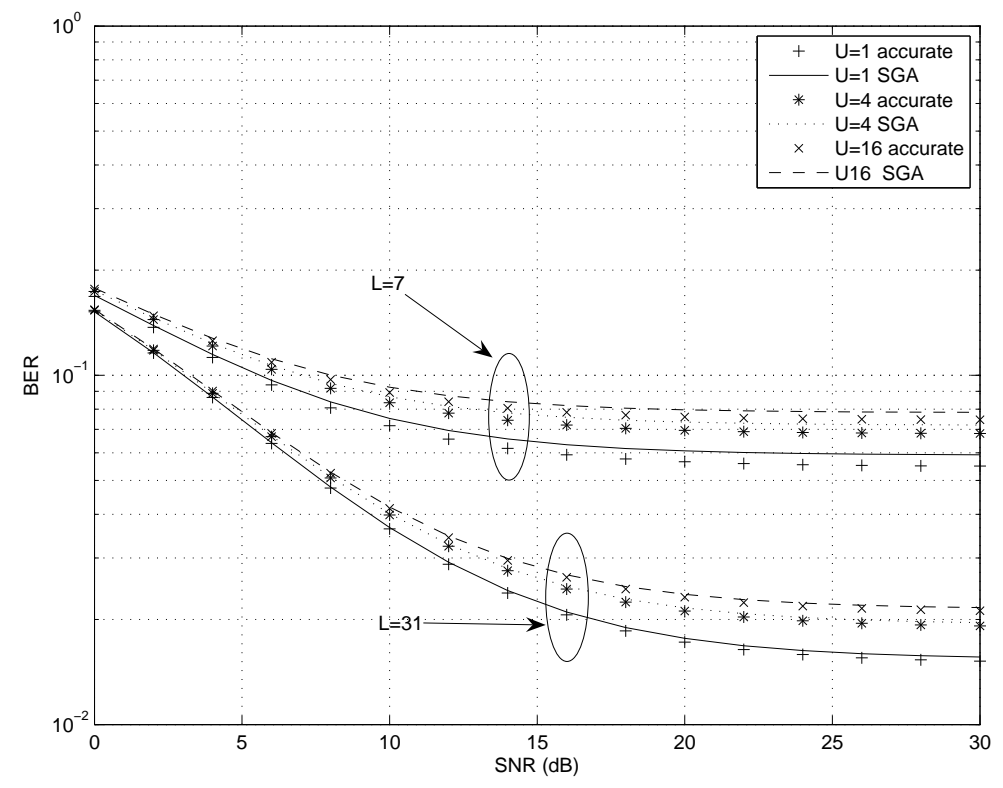

Figure 2. BER vs the per-bit SNR in an asynchronous MC-DS-CDMA exposed to Rayleigh fading with random spreading sequences and BPSK modulation. The length of the spreading sequence is $L=7$ and $L=31$. The number of subcarriers is $U=1,4$, and 9 . The average power of all subcarriers and users is equal. The number of users is $K=4$.

[2] L. Hanzo, T. Killer, M.S. Munster, and B.J. Choi, OFDM and MC-CDMA for Broadband Multiuser Communications, WLANs and Broadcasting, John Wiley \& Sons Inc, 2003.

[3] Y. Lie-Liang and L. Hanzo, "Performance of generalized multicarrier DS-CDMA over Nakagami-m fading channels," IEEE Transactions on Communications, vol. 50, no. 6, pp. 956-966, June 2002.

[4] S. Kondo, and L.B. Milstein, "Performance of multicarrier DS CDMA systems," IEEE Transactions on Communications, vol. 44, no. 2, pp. 238-246, Feb. 1996.

[5] M. O. Sunay, P. J. McLane, "Caculating error probabilities for DS-CDMA systems: when not to use the Gaussian approximation," Proc. IEEE GLOBECOM, vol. 3, London, UK, Nov. 1996, pp. 1744-1749.

[6] J. Cheng and N. C. Beaulieu, "Accurate DS-CDMA bit-error probability calculation in Rayleigh fading," IEEE Transactions on Wireless Communications, vol. 1, no. 1, pp. 315, Jan. 2002.

[7] J. Cheng and N. C. Beaulieu, "Precise bit error rate calculation for asynchronous DSCDMA in Nakagami fading," in Proc. IEEE GLOBECOM, San Francisco, CA, Nov. 2000, pp. 980-984.

[8] D. Liu, C. L. Despins, and W. A. Krzymien, "Low-complexity performance evaluation of binary and quaternary DS-SSMA over Rician fading channels via the characteristic function method," Wireless Personal Commun., vol. 7, pp. 257-273, Aug. 1998. 
[9] S. W. Oh and K. H. Li, "Performance evaluation for forward-link cellular DS-CDMA over frequency-selective Nakagami multipath fading channels," Wireless Personal Commun., vol. 18, pp. 275-287, Sep. 2001.

[10] B. Smida, C. L. Despins, and G. Y. Delisle, "MC-CDMA performance evaluation over a multipath fading channel using the characteristic function method," IEEE Transactions on Communications, vol. 49, no. 8, pp. 1325-1328, Aug. 2001.

[11] J. S. Lehnert and M. B. Pursley, "Error probabilities for binary direct-sequence spread spectrum communications with random signature sequences," IEEE Transactions on Communications, vol. 35, pp. 87-98, Jan. 1987.

[12] I. S. Gradshteyn and I.M. Ryzhik, I. M. Table of Integrals, Series, and Products, New York: Academic Press, Sixth edition, July 2000. 